\title{
Review
}

Bing Liu*

\section{Treatment of systemic diseases and oral focal infection}

DOI: 10.2478/ii-2018-0002

Received January 28, 2018; accepted February 06, 2018; published online April 10, 2018

\begin{abstract}
Oral lesions are highly correlated with the occurrence and development of many diseases. In addition, the treatment of systemic diseases may aggravate oral focal infections, affect the life quality of patients, interfere with the treatment of systemic diseases, and even cause systemic infection in serious cases. Treatment strategies for systemic diseases may induce or aggravate oral local lesion infections. In specific, administration of oral antiepileptic drugs and immunosuppressive drugs may induce gingivitis, radiotherapy or chemotherapy for malignant tumors may cause oral mucositis, long-term use of bisphosphonates for inhibition of tumor bone metastasis or prevention of osteoporosis may cause osteonecrosis of the jaw, and allogeneic hematopoietic stem cell transplantation may cause oral rejection reactions.
\end{abstract}

Keywords: oral focal infection, systemic diseases, drug gingivitis, malignant tumor

\section{Introduction}

Recent studies have shown that periodontitis is correlated with cardiovascular system diseases, diabetes, gastritis, bronchopneumonia, and premature and new-born infants with a low birth weight. The pathogenesis of periodontitis is related to the infection state of the oral focus [1-3]. The existence and treatment of some systemic diseases induce or aggravate oral focal infection. The occurrence of infection depends on two factors. One is the quality and quantity of pathogenic microbes (the type and number of bacteria in the lesion and the toxicity of pathogenic bacteria), and the other is the resistance and immune function of the body. The immune function of the body is reduced in patients with systemic diseases, leading to acute attack of the oral focus. Mild oral focal infection hinders the treatment of systemic diseases, but serious infection induces systemic diseases. Thus, the immune function may influence the life and health of patients. Treatment of some systemic diseases affects the local immune state of the oral cavity, causes local focal infection, and even induces systemic infection, leading to adverse effects on health. This article summarizes the treatment strategies for systemic diseases that cause or aggravate oral focal infection.

\section{Drug-induced gingivitis due to long-term use of oral drugs}

Drug-induced gingival hyperplasia refers to the increase in gingival fiber composition due to the longterm use of certain drugs, the most common of which include the antiepileptic drug phenytoin (Dilantin), cyclosporine, and the calcium channel antagonist nifedipine (Adalat). In vitro culture showed that phenytoin sodium stimulates the mitotic activity of fibroblasts, thereby promoting the synthesis of proteins and collagen. Meanwhile, cells secrete inactive collagenolytic enzymes. The synthesis of collagen is greater than

Department of Stomatology, Tianjin Medical University General Hospital, Tianjin 300052, China

${ }^{\star}$ Correspondence: Bing Liu, E-mail: BingLtj@163.com 
its degradation, leading to the proliferation of connective tissues. Recent studies have shown that the degree of gingival hyperplasia is not influenced by gender, dosage, time, and drug concentration in the serum and saliva but is related to the oral hygiene, original gingival inflammation, and age of patients [4]. Human and animal experiments have shown that if gingivitis is not caused by plaque and other stimulants, then druginduced hyperplasia can be greatly reduced or avoided. However, some studies have also reported that druginduced hyperplasia may occur without local stimulation. In addition, some cases of spontaneous gingival bleeding are caused by the oral use of warfarin. In addition to drugs, clotting disorders may be associated with the original inflammatory state of the periodontium [4].

\section{Oral symptoms caused by chemotherapy}

Approximately $40 \%$ of cancer patients who undergo chemotherapy suffer from oral mucositis. The immune function of the patients with cancer is damaged. The direct damage of the treatment to the oral and maxillofacial region can easily cause acute attacks of the original oral cavity or new oral complications. Radiotherapy combined with chemotherapy increases the risk and severity of oral mucositis. Several chemotherapeutic drugs can cause oral mucositis in patients with cancer. In particular, etoposide, methotrexate, irinotecan, and fluorouracil exert stronger toxicity to the mucous membrane compared with other chemotherapeutic drugs, thereby increasing the risk of developing severe mucositis [5]. Pico et al. [6] found that etoposide and methotrexate have direct mucosal toxicity and can be secreted in the saliva, which prove their significant oral mucosal toxicity. Studies have found that chemotherapeutic drugs prevent epithelial cells from differentiation and maturation, leading to mucous ulcer and atrophy [7] after 7-14 days of treatment initiation in $76.3 \%$ of patients [8-10]. Ruescher et al. [11] conducted a clinical study on 24 bone marrow transplant recipients undergoing high-dose chemotherapy and found that the possibility of hemolytic streptococcal bacteremia is three times higher in patients with oral mucositis than in controls. Streptococcus is the most common bacterium causing oral mucositis. Colonization of the dominant bacteria in the oral cavity also causes the infection. The opportunistic pathogen Blastomyces albicans can colonize the skin and oral mucosa of human beings. Healthy oral mucosa is an important barrier against fungal infection. While neutrophil granulocyte is reduced, long-term application of antibiotics or simultaneous use of glucocorticoids can create a favorable environment for fungal overgrowth. Low immune function or poor control of oral local fungal infection increases the risk of systemic fungal infection. Existing antifungal drugs may cause severe complications or even death. Therefore, early detection and treatment of local fungal infections are highly important.

After patients with hematologic malignancies receive systemic radiation therapy, myelosuppression and granulocytic reduction further decrease their immune function. Reduced number and function of phagocytes and $\mathrm{T}$ lymphocytes due to specific and nonspecific humoral immune factor deficiency lead to lack of specific antibodies on the mucosal surface, thereby increasing susceptibility to pathogenic microbes and oral focal infection [12]. In addition, radiotherapy for malignant tumors in the mouth can directly damage the oral mucosa. Radiation is more lethal to proliferating cells. With active proliferation, the oral mucosa is easily damaged during local radiotherapy; as a result, the mucosal mechanical barrier may be destroyed. The erosive and necrotic tissues of the oral mucosa provide a large amount of nutrients for the growth and metabolism of bacteria. Ulceration and latent lacunae reduce the redox potential, allowing anaerobes to colonize easily and cause infection [13]. Radiation can also induce microcirculatory vessel narrowing in the field of radiation or blockage of the radiation field. Thus, radiation causes congestion and edema of the local mucous membrane and leads to radioactive oral mucositis. Good oral hygiene condition prevents severe oral mucositis. The aerobic Gram-negative bacteria in the oral cavity of patients with good oral hygiene lack the conducive environment to proliferate. This condition causes the bacteria to release less toxins, thereby reducing the incidence of oral mucositis.

Radiotherapy and chemotherapy can cause different degrees of oral drying, next only to fatigue, sleepiness, and pain, as the most common symptoms of patients with tumor. The salivary glands are also in the same range of radiation in head and neck cancer patients receiving radiotherapy. The secretion of salivary glands after radiation is significantly reduced, inhibited, or changed. Signs of damage to the 
salivary glands by radiotherapy appear at the first week after radiotherapy. When the dose of radiotherapy exceeds 4000-5000 cGy, Zagari's disease may be permanent. Normal saliva flow plays an important role in cleaning and maintaining the balance of normal flora of the mouth. Saliva contains a variety of immune components, which can reduce the risk of infection. Decreased saliva in the mouth of patients increases risk of infection by cariogenic bacteria, leading to acid demineralization and even severe caries.

Compared with radiotherapy, chemotherapy does notinduce more significant oral drying, but somestudies have found that it may cause severe oral drying $[14,15]$. However, a few researchers determined the histological changes in salivary glands after chemotherapy. Jensen et al. [14] found that saliva flow significantly changes after chemotherapy. Lockhart and Sonis [15] reported that $50 \%$ of patients who receive chemotherapy during autopsy suffer from catheter dilatation, cystogenia, acinar degeneration, and inflammatory cell infiltration in glandular tissues. This finding suggests that chemotherapeutic drugs contribute to the destruction of salivary gland tissues. In addition to the direct destruction of salivary gland tissues, chemotherapy can also induce changes in saliva flow and salivary components, including sodium salt, magnesium salt, total protein, cell-growth factor, and immunoglobulin. Zagari's disease can lead to chewing and swallowing difficulties and dysphasia, increasing susceptibility to Candida infection, and severe caries.

Therefore, maintaining the oral health of patients with malignant tumor and leukemia receiving chemotherapy is essential. A good oral hygiene environment greatly reduces the incidence of oral complications. The corresponding oral treatment should also be very cautious. Treatment during the remission period is good to relieve pain and trauma and to reduce the stimulation of oral necrotic tissue. During chemotherapy induced myelosuppression and chemotherapy, exelcymosis, oral tissue biopsy, deep periodontal curettage, and other invasive treatments are not advisable. Even during remission, preventive oral treatments, including periodontal scaling, should be carried out carefully under common consultation of the physician. Any oral surgical treatment should be conservative. After receiving oral treatment, complications such as infection and bleeding should be closely observed. The application of alkaline gargle and antibiotics is of great significance in preventing fungal infection and alleviating necrotizing and ulcerative oral lesions.

\section{Bisphosphonates leading to osteonecrosis of the jaw}

Bisphosphonate is a new type of bone resorption inhibitor developed in the 1980s. At present, as a firstline drug, it is widely used in osteoclast-mediated bone diseases, such as osteoporosis, multiple myeloma, metastatic bone tumors, and scleromalacia (Paget's disease). However, with the wide application of these drugs, some new adverse reactions have emerged. In 2003, Marx [16] first reported that these drugs could induce bisphosphonate-related osteonecrosis of the jaw (BRONJ). The increasing number of case reports has attracted global attention. The occurrence of BRONJ is related to not only the duration of the use of bisphosphonates but also the route of administration. In 2004, Ruggiero et al. [17] found that the cumulative incidence of BRONJ by intravenous administration is $0.8 \%-12 \%$. They also revealed that intravenous administration could induce BRONJ and oral administration could cause dangerous risks. Oral invasive treatments are the common risk factors for developing BRONJ. For example, tooth extraction, dental implant surgery, apex surgery, and periodontal abscess increase the incidence of osteonecrosis [18]. Genes, drugs, and associated factors can also affect the occurrence of BRONJ. BONJ is clinically manifested as limited and nonfatal bone exposure within $>8$ weeks without history of local radiotherapy, which causes secondary infection due to local injury and results in acute osteomyelitis, dead bone formation, and extensive dental loosening [19]. BRONJ most often occurs in the jawbone ( $65 \%)$, followed by the supramaxilla $(\sim 26 \%)$; the simultaneous involvement of the jawbone and supramaxilla is rare $(\sim 9 \%)$.

The pathogenesis of BRONJ remains unclear. Relevant epidemiological evidence shows that its pathogenesis is influenced by time and dose. Several speculations about pathogenesis exist, but no strict causality has been reported.

BRONJ only occurs in the jaw, and the reconstruction rate of the jaw is much higher than those of other bones. The first consideration in the literature is that diphosphate inhibits the function of osteoclast, thereby 
affecting jaw bone reconstruction and angiogenesis. After alveolar surgery, the pathophysiological process of bone reconstruction cannot be completed and can cause osteonecrosis of the jaw [20]. Tumor patients receiving diphosphate treatment are at a high risk of developing BRONJ. Even if the same treatment is used to inhibit bone metastases, the incidence of BRONJ in patients with various tumors is greatly different. The incidence in patients with myeloma (55.9\%) is twice than in patients with breast cancer (33.4\%). Meanwhile, patients with prostate cancer have lower risk (4.6\%). Some studies have reported that other diseases such as arthritis, Crohn's disease, and osteodysplasty can increase the risk of BRONJ [20].

BRONJ is difficult to treat. Conservative treatment can relieve symptoms but cannot cure BRONJ. Surgical intervention can eliminate the infection thoroughly but can increase the risk of aggravating osteonecrosis. In addition, surgery should be performed when the general condition of the patient is tolerable. Therefore, the key is effective prevention. Oral invasive treatment is an important risk factor for the occurrence of BRONJ. Therefore, dental clinicians, before providing oral-related treatments, must pay attention to the whole-body health and systemic medication of patients to avoid BRONJ caused by trauma in the oral and maxillofacial region.

\section{Rejection reaction after allogeneic bone marrow transplantation}

Oral mucositis is common among patients with malignant hematological diseases who have been treated with myeloablative and hematopoietic stem cell transplantation. The incidence of oral mucositis in patients with bone marrow transplant is high (70\%-90\%) [21,22]. According to the time of rejection reaction, rejection reaction is considered acute (within 100 days after transplantation) or chronic (100 days after transplantation). Acute rejection reaction with severe manifestation has a high mortality rate. Chronic rejection reaction is manifested in autoimmune diseases, such as skin disease, liver dysfunction, pulmonary fibrosis, changes in the mucosa of the oral and digestive tracts, and drop of saliva and tear flow. Oral mucosal damage is the second major lesion following skin damage. Oral lesions account for 33\%-50\% of acute rejection and $60 \%-80 \%$ of chronic rejection [23,24]. Typical manifestations of the rejection in the mouth are moss-like damage, erythema, ulcer, dry mouth, mucocele, and occasionally observed pyogenic granuloma. These lesions should be identified with lichen planus, erythema polyformis, lupus erythematosus, pemphigus, scleroderma, and chemotherapy-associated lesions [25]. Treister et al. reported that $45 \%$ of children receiving bone marrow transplantation have oral lesions. Among these lesions, erythema accounting for $42 \%$ and moss-like lesion accounting for $36 \%$ are two major symptoms [26].

For long-term surviving bone marrow transplant patients, typical rejection reactions, secondary malignant tumors, and lymphoproliferative diseases are also common after bone marrow transplantation. The risk of these patients suffering from malignant tumors, such as oral squamous cell carcinoma, is 13 times more than that of ordinary people [27-29]. Moreover, the application of a large number of immunosuppressive agents, radiotherapy, and chemotherapy increases the probability of opportunistic infection. The most common agent of opportunistic infection in patients with bone marrow transplantation is Candida. Related dangerous risk factors include decreased saliva flow, saliva Immunoglobulin A (IgA) concentration, and neutrophils. Epstein et al. [29] found oral Candida colonization in 31\% of patients with bone marrow transplantation. Of these patients, 56\% exhibited clinic symptoms. In patients with abnormal salivary gland function, acute pseudomembranous-type Candida stomatitis is the most common. Preventive use of fluconazole or chlorhexidine gargle may help reduce oral colonization and may reduce the possibility of infection throughout the body.

The mouth is a highly complex and special microecosystem in which $>1000$ microbes with $200-300$ types are present [30]. The mouth is a base camp of chronic inflammation of the human body. Gingivitis, periodontitis, chronic pulpitis, periapical periodontitis, pericoronitis, and residual root disease are common in the population. Most oral lesions, especially chronic periodontitis, have no enough apparent pain to be alerted. Therefore, timely treatment is difficult. In the case of normal immune function, oral lesions exist for a long time without attack. Once the immune function changes, an acute attack may occur, even inducing 
infection during the treatment of certain systemic diseases. Thus, treatment plans for systemic diseases are difficult to proceed smoothly. This phenomenon affects the quality of life of patients and endangers their entire health. Stomatology doctors need to pay close attention to the advancement of medical development to understand the progress of the treatment of systemic diseases and the adverse reactions of drugs. General practitioners should have an overall concept, pay attention to the oral health of patients, deal with oral lesions before treatment of system diseases, and design a treatment plan suitable for these patients.

Acknowledgments: None.

Conflict of interest: The author states no conflicts of interest.

\section{References}

[1] DeStefano F., Anda R.F., Kahn H.S., Williamson D.F., Russell C.M., Dental disease and risk of coronary heart disease and mortality, Clin. Res., 1993, 306(6879), 688-691.

[2] Kiran M., Arpak N., Unsal E., Erdoğan M.F., The effect of improved periodontal health on metabolic control in type 2 diabetes mellitus, J. Clin. Periodontol., 2005, 32(3), 266-272.

[3] Offenbacher S., Katz V., Fertik G., Collins J., Boyd D., Maynor G., et al., Periodontal infection as a possible risk factor for preterm low birth weight, J. Periodontol., 1996, 67(10 Suppl.), 1103-1113.

[4] Cawley M.M., Benson L.M., Current trend in managing oral mucositis clinical, Clin. J. Oncol. Nurs., 2005, 9(5), 584-592.

[5] Epstein J.B., Tsang A.H., Warkentin D., Ship J.A., The role of salivary function in modulating chemotherapy-induced oropharyngeal mucositis: a review of the literature, Oral Surg. Oral Med. Oral Pathol. Oral Radiol. Endod., 2002, 94(1), 39-44.

[6] Pico J.L., Avila-Garavito A., Naccache P., Mucositis: its occurrence, consequences, and treatment in the oncology setting, Oncologist, 1998, 3(6), 446-451.

[7] Peterson D.E., Oral toxicity of chemotherapeutic agents, Semin Oncol., 1992, 19(5), 478-491.

[8] Puyalt Casado M., Jiménez Martinez C., Chimenos Küstner E., López López J., Juliá A., A protocol for the evaluation and treatment of oral mucositis in patients with hematological malignancies, Med. Oral., 2003, 8(1), 10-18.

[9] López J., Sabater M.M., Munoz J., Rosello X., Granena A., Evaluation and prevention of oral complications in patients subjected to bone marrow transplantation: a clinical study, Med. Oral., 2000, 5(5), 345-354.

[10] Woo S.-B., Sonis S.T., Monopoli M.M., Sonis A.L., A longitudinal study of oral ulcerative mucositis in bone marrow transplant recipients, Cancer, 1993, 72(5), 1612-1617.

[11] Ruescher T.J., Sodeifi A., Scrivani S.J., Kaban L.B., Sonis S.T., The impact of mucositis on alpha-hemolytic streptococcal infection in patients undergoing autologous bone marrow transplantation for hematologic malignancies, Cancer, 1998, 82(11), 2275-2281.

[12] Nagy K.N., Sonkodi I., Szöke I., Nagy E., Newman H.N., The microflora associated with human oral carcinomas, Oral Oncol., 1998, 34(4), 304-308.

[13] Dörr W., Hamilton C.S., Boyd T., Reed B., Denham J.W., Radiation-induced changes in cellularity and proliferation in human oral mucosa, Int. J. Radiat. Oncol. Biol. Phys., 2002, 52(4), 747-911.

[14] Jensen S.B., Pedersen A.M., Reibel J., Nauntofte B., Xerostomia and hypofunction of the salivary glands in cancer therapy, Support. Care Cancer, 2003, 11(4), 207-225.

[15] Lockhart P.B., Sonis S.T., Alterations in the oral mucosa caused by chemotherapeutic agents: a histologic study, J. Dermatol. Surg. Oncol., 1981, 7(12), 1019-1025.

[16] Marx R.E., Pamidronate (Aredia) and zoledronate (Zometa) induced a vascular necrosis of the jaws: a growing epidemic, J. Oral Maxillofac. Surg., 2003, 61(9), 1115-1117.

[17] Ruggiero S.L., Dodson T.B., Assael L.A., Landesberg R., Marx R.E., Mehrotra B., et al., American Association of Oral and Maxillofacial Surgeons position paper on bisphosphonate-related osteonecrosis of the jaw - 2009 update, Aust. Endod. J., 2009, 35(3), 119-130.

[18] Marx R.E., Cillo J.E. Jr., Ulloa J.J., Oral bisphosphonate-induced osteonecrosis: risk factors, prediction of risk using serum CTX testing, prevention, and treatment, J. Oral Maxillofac. Surg., 2007, 65(12), 2397-2410.

[19] Migliorati C.A., Siegel M.A., Elting L.S., Bisphosphonate-associated osteonecrosis: a long-term complication of bisphosphonate treatment, Lancet Oncol., 2006, 7(6), 508-514.

[20] Allen M.R., Burr D.B., The pathogenesis of bisphosphonate-related osteonecrosis of the jaw: so many hypotheses, so few data, J. Oral Maxillofac. Surg., 2009, 67(5 Suppl.), 61-70.

[21] Ratanatharathorn V., Ayash L., Lazarus H.M., Fu J., Uberti J.P., Chronic graft-versus-host disease: clinical manifestation and therapy: mini review, Bone Marrow Transplant., 2001, 28(2), 121-917. 
[22] Nicolatou-Galitis O., Kitra V., Van Vliet-Constantinidou C., Peristeri J., Goussetis E., Petropoulos D., et al., The oral manifestations of chronic graft-versus-host disease (cGVHD) in pediatric allogeneic bone marrow transplant recipients, J. Oral Pathol. Med., 2001, 30(3), 148-153.

[23] Barret A.P., Bilous A.M., Oral patterns of acute and chronic graft-v-host disease, Arch. Dermatol., 1984, 120(11), $1461-1465$.

[24] Shubert M.M., Sullivan K.M., Morton T.H., Izutsu K.T., Peterson D.E., Flournoy N., et al., Oral manifestations of chronic graft-v-host disease, Arch. Intern. Med., 1984, 144(8), 1591-1595.

[25] Eggleston T.I., Ziccardi V.B., Lumerman H., Graft-versus-host disease: case report and discussion, Oral Surg. Oral Med. Oral Pathol. Oral Radiol. Endod., 1998, 86(6), 692-696.

[26] Treister N.S., Woo S.B., O’Holleran E.W., Lehmann L.E., Parsons S.K., Guinan E.C., et al., Oral chronic graft-versus-host disease in pediatric patients after hematopoietic stem cell transplantation, Biol. Blood Marrow Transplant., 2005, 11(5), 721-731.

[27] Demarosi F., Lodi G., Carrassi A., Soligo D., Sardella A., Oral malignancies following HSCT: graft-versus-host disease and other risk factors, Oral Oncol., 2005, 41(9), 865-877.

[28] Curtis R.E., Rowlings P.A., Deeg H.J., Shriner D.A., Socíe G., Travis L.B., et al., Solid cancers after bone marrow transplantation, N. Engl. J. Med., 1997, 336(12), 897-904.

[29] Epstein J.B., Raber-Durlacher J.E., Wilkins A., Chavarria M.G., Myint H., Advances in hematologic stem cell transplant: an update for oral health care providers, Oral Surg. Oral Med. Oral Pathol. Oral Radiol. Endod., 2009, 107(3), 301-312.

[30] Paster B.J., Boches S.K., Galvin J.L., Ericson R.E., Lau C.N., Levanos V.A., et al., Bacterial diversity in human subgingival plaque, J. Bacteriol., 2001, 183(12), 3770-3783. 